\title{
Nonlinear Normal Mode continuation through a Proper Generalized Decomposition approach with modal enrichment
}

\author{
L. Meyrand ${ }^{\mathrm{a}}$, E. Sarrouy ${ }^{\mathrm{a}, *}$, B. Cochelin ${ }^{\mathrm{a}}$, G. Ricciardi ${ }^{\mathrm{b}}$ \\ ${ }^{a}$ Aix Marseille Univ, CNRS, Centrale Marseille, LMA, Marseille, France \\ ${ }^{b}$ CEA Cadarache DEN/DTN/STCP/LHC, 13108 Saint-Paul-Lez-Durance Cedex, France
}

\begin{abstract}
Nonlinear normal modes (NNMs) of a mechanical structure provide a mean to understand nonlinear vibrational phenomena measured experimentally. As they are an interesting extension of the linear normal modes (LNMs), numerical methods are needed to estimate these oscillatory motions, generally including continuation aspects with respect to the circular frequency. In this paper, a continuation method combined to a model reduction based on Proper Generalized Decomposition (PGD) technique is described. This formulation mixes PGD, harmonic balance method (HBM) and basic continuation techniques in order to reach a highly reduced description of the NNMs. This PGD/HBM-based continuation algorithm includes a modal enrichment as the NNM energy grows. The method is applied to two conservative problems including a cubic spring and a unilateral contact.
\end{abstract}

Keywords: Continuation, Model reduction, Proper Generalized

Decomposition (PGD), Nonlinear dynamics, Nonlinear Normal Mode, Harmonic Balance Method (HBM)

\footnotetext{
${ }^{*}$ Corresponding author

Email addresses: meyrand@lma.cnrs-mrs.fr (L. Meyrand), emmanuelle.sarrouy@centrale-marseille.fr (E. Sarrouy), bruno.cochelin@centrale-marseille.fr (B. Cochelin), guillaume.ricciardi@cea.fr (G. Ricciardi)
} 


\section{Introduction}

In the frame of nonlinear dynamics, the Nonlinear Normal Modes (NNMs) have been developed for several decades, in the aim to use them as their wellknown linear counterparts (LNMs for Linear Normal Modes). Their properties 5 are investigated in order to enable faster computations using modal reduction and to ease the forced dynamics prediction and interpretation [1, 2]. Basically, NNMs are the sets of periodic solutions of an unforced and - generally - undamped mechanical system $[3,4,5]$. The last decade witnessed the development of numerical methods dedicated to NNMs but this research area has not yet reached maturity $[6,5]$. Although algorithms can now be effectively applied to real engineering structures, the discrete systems to solve are often high-dimensional problems and their nonlinear contribution causes convergence issues: NNMs require large sets of variables to be properly described which make them hard to compute in short times and hard to reuse even if some works still 15 take advantage of their computation [7].

Model reduction techniques have taken an important place in the solving process of mechanical systems, as their numerical complexity is increasing and the models are becoming more and more detailed and realistic. The continuation of NNMs using reduced-order models has already been addressed, for instance in

$20[8,9]$. Both these works use a shooting method combined to a pseudo-arclength continuation. Model reduction comes from the space where the $\mathbf{q}_{0}$ point from which time integration starts is looked for. Kuether and Allen [8] build this reduced space by using either a set of LNMs directly or the static displacement resulting from a force based on a set of LNMs while Sombroek et al. [9] use 25 some LNMs and their modal derivatives.

This paper proposes to compute NNMs using a Proper Generalized Decomposition (PGD) approach combined to a continuation method. The PGD methods are a class of reduction techniques based on the separation of the unknowns which allows dealing with smaller systems to reduce the algorithmic 
been detailed by Chinesta in [10] and the literature shows that a wide range of problems can be managed with this approach [11, 12, 13]. PGD was already used by Grolet and Thouverez [14] to compute free and forced response of nonlinear systems. The interest of the paper is first to couple this approach to a continuation method with a progressive enrichment of the PGD modes family and second to improve some of the PGD steps to get a robust algorithm. Contrarily to methods proposed in $[8,9]$, the reduced space is not built using $a$ priori quantities but on the fly. The resulting method is applied to two simple systems which allows for an accurate analysis of its performance. The detailed study of the physical meaning of NNMs and their internal resonances or their use for the computation of forced responses is beyond the scope of this article. The reader is referred to $[15,16,7]$ for detailed works on theses aspects.

The paper is organized as follows: the mechanical framework is described in Sec. 2, including a short review of the NNM definition. In Sec. 3 the PGD and

45 the Harmonic Balance Method (HBM) frameworks are exposed. The differences with Grolet and Thouverez [14] work are highlighted. Then the PGD/HBMbased continuation is described in Sec. 4. This part is a proposition of a full algorithm to compute NNMs with on the fly enrichment of the reduced basis in conjunction with continuation techniques. The last section, Sec. 5 is dedicated to two illustrative cases.

\section{Mechanical framework}

This section first describes the general equations of a nonlinear dynamical problem and then defines the associated NNMs.

\subsection{Nonlinear dynamical system}

After a spatial discretisation, using for example the Finite Elements method, the differential equations governing the motion of a nonlinear dynamical system can usually take the following form $[17,7]$, where the nonlinear efforts are 
separated from the linear ones:

$$
\mathbf{M} \ddot{\mathbf{u}}+\mathbf{C} \dot{\mathbf{u}}+\mathbf{K u}+\mathbf{f}_{\mathrm{nl}}(\mathbf{u}, \dot{\mathbf{u}})=\mathbf{f}(t)
$$

Eq. (1) is a set of $N$ second order nonlinear differential equations, the vector of $\mathbb{R}^{N}$. $\mathbf{M}, \mathbf{C}$ and $\mathbf{K}$ are respectively the linear mass, damping and stiffness matrices, with $\{\mathbf{M}, \mathbf{C}, \mathbf{K}\} \in \mathcal{M}_{N}(\mathbb{R})^{3}$. $\mathbf{f}(t)$ is the vector of excitation forces and lies in $\mathbb{R}^{N}$ too. Finally $\mathbf{f}_{\mathrm{nl}}(\mathbf{u}, \dot{\mathbf{u}})$ is the vector of nonlinear forces and belongs to $\mathbb{R}^{N}$.

65 mechanical problems. Indeed, the $\mathbf{f}_{\mathrm{nl}}$ term can be any nonlinear expression (e.g. cosine function, polynomial terms like $u_{i} u_{j}^{3}$, non smooth function, etc.).

\subsection{Short review of nonlinear normal modes}

This paper focuses on the computation of nonlinear normal modes. NNMs can be defined as the periodic solutions of Eq. (1) in the particular case when no forcing neither damping is considered or as an extension of the definition of linear normal modes which takes into account the nonlinear vector $\mathbf{f}_{\mathrm{nl}}$. Multiple definitions of NNMs can be found in the literature: NNMs were introduced by Rosenberg in the 1960's [3] for conservative systems as a periodic vibration in 75 which all the degrees of freedom are synchronous (same origin, same extrema, same period) and can be expressed from only one of them. Shaw and Pierre $[18,4]$ extended this definition in the 1990's to all the autonomous systems, including the dissipative ones. A NNM is then defined as a two-dimensional invariant manifold in phase space. The reader can find more information about

NNMs definitions in the works of Kerschen and Renson [19, 17, 5] for instance. More recently, Haller and Ponsioen [20] introduced a new definition for NNMs and the notion of spectral submanifolds.

Assuming the Rosenberg framework - that is undamped modes - a NNM is a set of limit cycles - or periodic solutions - of the following system:

$$
\mathbf{M} \ddot{\mathbf{u}}+\mathbf{K u}+\mathbf{f}_{\mathrm{nl}}(\mathbf{u})=\mathbf{0}
$$


To shorten further expressions, a signal $\mathbf{u}(t)$ satisfying Eq. (2) will be equivalently denoted:

$$
\mathbf{R}(\mathbf{u}(t))=\mathbf{0}
$$

Although they do not have orthogonality properties like LNMs and do not decouple the equations, NNMs can be useful to investigate the modal interaction between widely spaced modes or modal bifurcations $[17,5]$. One of the major features of NNMs is that frequencies depends on the response amplitude on the contrary to LNMs.

According to the size and the complexity of the dynamical system studied, computing branches of NNMs can be expensive in terms of data storage and computation times [5]. This observation motivates the developments described in the rest of the paper.

\section{PGD and HBM combination to describe periodic solutions}

A HBM framework is interesting as NNMs are periodic solutions. Despite a truncation order dependence, it allows dealing with algebraic equations in the frequency domain instead of (nonlinear) ordinary differential equations in the time domain. However a simple HBM becomes costly as the number of dof increases and more harmonics are needed to accurately describe the behaviour of a system. A combination with a PGD reduction technique allows to compute smaller systems without losing HBM advantages.

In this section the HBM framework used to compute periodic solutions is first given. Then PGD principles are introduced: PGD temporal and spatial subproblems are defined in combination with the HBM. Finally the full PGD/HBM algorithm is described.

\subsection{Harmonic Balance Method}

Unlike shooting methods $[21,6]$, the HBM provides a mean to compute limit cycles without the need of a time integration scheme. Only main steps are briefly described here, introducing some notations which are later used. The 
reader is referred to the numerous works on this subject for detailed explanations $[22,23,24,25]$.

\subsubsection{Basic principle}

HBM is based on a Galerkin method: looking for a periodic signal $\mathbf{u}(t)$ with period $T=2 \pi / \omega$, a truncated Fourier series is used as a parametrization:

$$
\mathbf{u}(t)=\frac{\mathbf{a}_{0}}{\sqrt{2}}+\sum_{k=1}^{H}\left(\mathbf{a}_{k} \cos (k \omega t)+\mathbf{b}_{k} \sin (k \omega t)\right)
$$

where $\mathbf{a}_{k}$ and $\mathbf{b}_{k}$ are respectively the cosine and sine coefficients of the Fourier series. This decomposition also provides expressions for any $\mathbf{u}$ derivative with respect to time. Injecting this decomposition in the equations governing $\mathbf{u}$ and its derivatives (i.e. Eq. (1) or Eq. (2)) and following the Galerkin procedure, the equations are projected onto each element of the Fourier basis $[1 / \sqrt{2}, \cos (\omega t)$, $\sin (\omega t), \ldots, \cos (H \omega t), \sin (H \omega t)]$ using the inner product:

$$
<f, g>_{T}=\frac{2}{T} \int_{0}^{T} f(t) g(t) d t
$$

This leads to an algebraic set of $N(2 H+1)$ equations where $N$ is the size of the time problem; the unknowns are $\mathbf{a}_{k}$ and $\mathbf{b}_{k}$ vectors and sometimes $\omega$ when there is no external forcing (e.g. Eq. (2)). These equations are denoted:

$$
\mathbf{H}\left(\mathbf{a}_{0}, \mathbf{a}_{1}, \mathbf{b}_{1}, \ldots, \omega\right)=\mathbf{0}
$$

When the system has an external periodic forcing, that is when it is non autonomous, $\omega$ in the Fourier series is taken equal to the forcing circular frequency $\omega^{*}$; the resulting system, with $\omega=\omega^{*}$, is then square and has, locally, one solution only. In the case of NNMs computation, the system is autonomous and for a given $\omega=\omega^{*}$ value, although the system is still square, there is, locally, not only one solution but a family of solutions. An additional equation usually called phase condition [26] is then used to choose one solution among this family. Possible phase conditions and their consequences on the problem size are mentioned later in Sec. 3.1.3. 


\subsubsection{AFT method to get nonlinear forces Fourier decomposition}

The expression of the projection of the linear part of Eq. (1) or (2) can be established analytically [25]. When considering Eq. (2), it is $\mathbf{H}_{1}(\omega) \mathbf{u}_{H}$, where:

$$
\left\{\begin{array}{l}
\mathbf{H}_{1}(\omega)=\operatorname{diag}\left(\boldsymbol{\Lambda}_{0}, \boldsymbol{\Lambda}_{1}, \ldots, \boldsymbol{\Lambda}_{H}\right) \\
\boldsymbol{\Lambda}_{0}=\mathbf{K}, \boldsymbol{\Lambda}_{k}=\operatorname{diag}\left(\mathbf{K}-(k \omega)^{2} \mathbf{M}, \mathbf{K}-(k \omega)^{2} \mathbf{M}\right) \\
\mathbf{u}_{H}=\left\{\mathbf{a}_{0}^{\mathrm{T}}, \ldots, \mathbf{a}_{k}^{\mathrm{T}}, \mathbf{b}_{k}^{\mathrm{T}}, \ldots\right\}^{\mathrm{T}}
\end{array}\right.
$$

The difficult and time consuming step when solving this system is the eval-

130

uation of the projections of nonlinear forces $\mathbf{f}_{\text {nl }}(\mathbf{u}, \dot{\mathbf{u}})$ onto the Fourier basis, denoted $\mathbf{H}_{\mathrm{nl}}\left(\mathbf{u}_{H}, \omega\right)$. Unless an analytical expression can be derived, the most common approach is the Alternating Frequency/Time (AFT) method introduced by Cameron and Griffin [27]. $\mathbf{u}\left(t_{k}\right)$ and $\dot{\mathbf{u}}\left(t_{k}\right)$ are evaluated for specific $t_{k}$ values in $[0, T]$ using an Inverse Fast Fourier Transform (IFFT) based on $\mathbf{a}_{k}$ and $\mathbf{b}_{k}$ coefficients. Then, $\mathbf{f}_{\mathrm{nl}}\left(\mathbf{u}\left(t_{k}\right), \dot{\mathbf{u}}\left(t_{k}\right)\right)$ is computed for each time $t_{k}$; finally, a Fast Fourier Transform (FFT) is used to evaluate the projections, that is the coefficients of the Fourier series of $\mathbf{f}_{\mathrm{nl}}(\mathbf{u}, \dot{\mathbf{u}})$.

The algebraic nonlinear system to solve writes:

$$
\mathbf{H}_{\mathrm{l}}(\omega) \mathbf{u}_{H}+\mathbf{H}_{\mathrm{nl}}\left(\mathbf{u}_{H}, \omega\right)=\mathbf{0}
$$

\subsubsection{Phase condition when circular frequency is unknown}

When considering an autonomous system such as Eq. (2), no "initial time" exists and using $\mathbf{u}(t), t \in[0, T]$ or $\mathbf{u}\left(t^{\prime}+t\right), t^{\prime} \in \mathbb{R}$ to describe the limit cycle are equivalent. Hence, the Fourier coefficients are not uniquely defined and it is preferable to add an equation, frequently called phase condition so as to define a unique solution line of Eq. (8). Different equations can be added: the amplitude or velocity of a given dof can be imposed when $t=0$ in Eq. (4) [26]. This effectively adds an equation linking Fourier coefficients of this dof together. This may imply that solutions violating this condition will not be found. Another solution is to nullify one of the Fourier coefficients components; this is a priori of a lower cost as it removes one unknown [28]. Moreover, considering a conservative system with no symmetry, choosing to nullify one 
size is divided by almost 2 , having only to solve for $(H+1) \mathbf{a}_{k}$ coefficients only based on the $N(H+1)$ equations provided by projection on cosine functions only. Other equations are of course possible such as the CHBM developped by Coudeyras et al. [29] which involves the eigenvalues analysis of the tangent system or the integral condition used in AUTO [30].

When adding an equation, the resulting system (6) for an arbitrarily chosen $\omega^{*}$ value is not square anymore: the $N(2 H+1)$ Fourier coefficients are described by the $N(2 H+1)+1$ equations (HBM equations plus phase condition). There are different ways to circumvent this problem as stated in [5].

The rest of the paper focuses on undamped NNMs which can be described by a collection of cosine coefficients only. Systems with out-of-unison behaviour as defined by Hill et al. [31] are excluded. This is equivalent to a phase condition imposing a null velocity at initial time for one dof for systems without symmetries. Using this assumption, all the equations derived from the projection onto sine functions $\sin (k \omega t)$ are useless and then removed. Equation (8) still holds but with the following definitions replacing definitions given in Eq. (7):

$$
\left\{\begin{array}{l}
\mathbf{H}_{\mathbf{l}}(\omega)=\operatorname{diag}\left(\boldsymbol{\Lambda}_{0}, \boldsymbol{\Lambda}_{1}, \ldots, \boldsymbol{\Lambda}_{H}\right) \\
\boldsymbol{\Lambda}_{0}=\mathbf{K}, \boldsymbol{\Lambda}_{k}=\mathbf{K}-(k \omega)^{2} \mathbf{M} \in \mathcal{M}_{N}(\mathbb{R}) \\
\mathbf{u}_{H}=\left\{\mathbf{a}_{0}^{\mathrm{T}}, \ldots, \mathbf{a}_{H}^{\mathrm{T}}\right\}^{\mathrm{T}}
\end{array}\right.
$$

For a given $\omega^{*}$ value, the system is then square, having $N(H+1)$ unknowns $\left(\mathbf{a}_{0}, \mathbf{a}_{1}, \ldots, \mathbf{a}_{H}\right)$ driven by $N(H+1)$ equations (projections of Eq. (2) onto $[1 / \sqrt{2}, \cos (\omega t), \ldots, \cos (H \omega t)]$ functions $)$.

\section{2. $P G D$ combined to $H B M$}

According to Chinesta et al. [12] and Grolet and Thouverez [14], a PGD process can simply be reduced to three fundamental steps: separating variables, obtaining as many subproblems as there are variables, and run an alternated directions algorithm which solves each subproblem with the other variables which are fixed, in a same loop iteration. In this section the temporal and the spa- 
tial problems are obtained and coupled with HBM features to produce a global PGD/HBM algorithm.

\subsubsection{Separable variables assumption}

As a first PGD requirement, we choose to look for a specific form of solutions including a space-time separation. $\mathbf{u}(t)$, solution of Eq. (2), is sought as:

$$
\mathbf{u}(t) \approx \sum_{j=1}^{m} \mathbf{p}_{j} q_{j}(t) \Leftrightarrow \mathbf{u}(t) \approx \mathbf{P} \mathbf{q}(t) \text { with } \mathbf{P}=\left[\mathbf{p}_{1}, \ldots, \mathbf{p}_{m}\right]
$$

where $m$ is a positive integer such as $m<<N$ and denotes the number of PGD modes used for $\mathbf{u}$ decomposition, $\mathbf{P} \in \mathcal{M}_{N, m}(\mathbb{R})$ is the matrix of the $m$ PGD mode shapes $\mathbf{p}_{j}$, and $\mathbf{q}(t) \in \mathbb{R}^{m}$ is the vector containing the time dependence of each PGD mode. As a product of two data is involved, the spatial modes $\mathbf{p}_{j}$ are normalized to 1 arbitrarily; amplitude information is then reflected by $q_{j}(t)$ only.

One of the main advantages of the PGD approach is to split the problem into two smaller problems: one in space returning a value of $\mathbf{P}$ and one in time to get $\mathbf{q}$. As we are here looking for periodic solutions, $\mathbf{q}$ will be decomposed using HBM. Following Sec. 3.1, $\mathbf{q}(t)$ is approximated by its truncated Fourier series:

$$
\mathbf{q}(t)=\frac{\mathbf{a}_{0}}{\sqrt{2}}+\sum_{k=1}^{H} \mathbf{a}_{k} \cos (k \omega t)
$$

A compact way to write $\mathbf{q}(t)$ is then:

$$
\mathbf{q}(t)=\mathbf{Q}_{H} \mathbf{h}_{H}(t, \omega), \text { where }\left\{\begin{array}{l}
\mathbf{Q}_{H}=\left[\mathbf{a}_{0}, \mathbf{a}_{1}, \ldots\right] \\
\mathbf{h}_{H}=[1 / \sqrt{2}, \cos (\omega t), \ldots]^{\mathrm{T}}
\end{array}\right.
$$

Computing $\mathbf{q}(t)$ is then equivalent to computing both $\mathbf{Q}_{H}$ and $\omega$. For examples of problems solved by PGD in the time domain, the reader can refer to Nouy [13] for instance.

\subsubsection{Temporal problem $\mathcal{T}_{m}$}

In this part, the mode shapes $\mathbf{P}$ are assumed to be known. The objective is to compute $\mathbf{q}(t)$ - i.e. $\mathbf{Q}_{H}$ and $\omega$ - with $\mathbf{P}$ as a parameter. To this end, a weak 
formulation is used: the test function $\mathbf{u}^{\star}(t)$ is written $\mathbf{u}^{\star}(t)=\mathbf{P} \mathbf{q}^{\star}(t)$ and $\mathbf{q}(t)$ must satisfy Eq. (13) for an oscillation period $I_{t}=[0,2 \pi / \omega]$.

$$
\forall t \in I_{t} \quad \forall \mathbf{q}^{\star}(t) \int_{I_{t}} \mathbf{q}^{\star T}(t) \mathbf{P}^{T} \mathbf{R}(\mathbf{P q}(t)) d t=0
$$

A few matricial calculus are needed to get the temporal problem (14) from the weak formulation (13):

$$
\forall t \in I_{t} \quad \mathbf{M}_{r} \ddot{\mathbf{q}}(t)+\mathbf{K}_{r} \mathbf{q}(t)+\mathbf{f}_{\mathrm{nl} r}(\mathbf{P q}(t))=\mathbf{0}
$$

where $\left\{\mathbf{M}_{r}=\mathbf{P}^{T} \mathbf{M P}, \mathbf{K}_{r}=\mathbf{P}^{T} \mathbf{K} \mathbf{P}\right\} \in \mathcal{M}_{m}(\mathbb{R})^{2}$ and $\mathbf{f}_{\mathrm{nl} r}=\mathbf{P}^{T} \mathbf{f}_{\mathrm{nl}} \in \mathbb{R}^{m}$.

This subproblem is a system of $m$ second order ODEs with a nonlinear contribution solved using HBM as described in Sec. 3.1. A new algebraic system with $m(H+1)$ equations and $m(H+1)+1$ unknowns - $\mathbf{Q}_{H}$ and $\omega-$ is then obtained. Indeed $\omega$ is unknown for a NNM computation and a constraint equation $c\left(\mathbf{q}_{H}, \omega\right)$ is needed to make the system square. Using the notations introduced in Eq. (8) and (9), the temporal problem $\mathcal{T}_{m}$ can be defined as follows:

$$
\mathcal{T}_{m}\left(\mathbf{Q}_{H}, \omega \mid \mathbf{P}\right)=\mathbf{0} \Leftrightarrow\left\{\begin{array}{l}
\mathbf{H}_{l}(\omega) \mathbf{q}_{H}+\mathbf{H}_{\mathrm{nl}}\left(\mathbf{q}_{H}, \omega\right)=\mathbf{0} \\
c\left(\mathbf{q}_{H}, \omega\right)=0
\end{array}\right.
$$

185

where $\mathbf{q}_{H}=\left\{\mathbf{a}_{0}^{\mathrm{T}}, \mathbf{a}_{1}^{\mathrm{T}}, \ldots\right\}^{\mathrm{T}}$ contains the columns of $\mathbf{Q}_{H}, \mathbf{H}_{1}$ is the linear contribution, $\mathbf{H}_{\mathrm{nl}}$ is the vector of Fourier coefficients of the nonlinear contribution of Eq. (14), and $c\left(\mathbf{q}_{H}, \omega\right) \in \mathbb{R}$ is an arbitrary constraint equation which allows to get a square system. $c\left(\mathbf{q}_{H}, \omega\right)$ will be specified in Sec. 4.1 as a continuation criterion.

\subsubsection{Spatial problem $\mathcal{S}_{m}$}

In this part, $\mathbf{q}(t)$ - that is $\mathbf{Q}_{H}$ and $\omega$ - are supposed to be known and only $\mathbf{P}$ is modified. The test function is then $\mathbf{u}^{\star}=\sum_{k=1}^{m} \mathbf{p}_{k}^{\star} q_{k}$ which leads to a set of $m$ systems with $N$ equations:

$$
\forall k \in \llbracket 1 ; m \rrbracket, \sum_{j=1}^{m}\left(\int_{I_{t}} q_{k} \ddot{q}_{j} d t \mathbf{M}+\int_{I_{t}} q_{k} q_{j} d t \mathbf{K}\right) \mathbf{p}_{j}+\int_{I_{t}} q_{k} \mathbf{f}_{\mathrm{nl}}(\mathbf{P q}(t)) d t=\mathbf{0}
$$


This can be written in a more compact way as a unique $N \times m$ sized system defining $\mathcal{S}_{m}$ :

$$
\mathcal{S}_{m}\left(\mathbf{P} \mid \mathbf{Q}_{H}, \omega\right)=\mathbf{0} \Leftrightarrow \mathbf{S}_{1} \tilde{\mathbf{p}}+\mathbf{S}_{\mathrm{nl}}(\tilde{\mathbf{p}})=\mathbf{0}
$$

where

- $\tilde{\mathbf{p}}=\left\{\mathbf{p}_{1}^{\mathrm{T}}, \ldots, \mathbf{p}_{m}^{\mathrm{T}}\right\}^{\mathrm{T}} \in \mathbb{R}^{N \times m}$ contains the columns of $\mathbf{P}$

- $\mathbf{S}_{1}$ can be defined analytically:

$$
\mathbf{S}_{1}=\mathbf{I}_{2} \otimes \mathbf{M}+\mathbf{I}_{0} \otimes \mathbf{K}
$$

$$
\text { with } \mathbf{I}_{0}=\frac{\pi}{w} \mathbf{Q}_{H} \mathbf{Q}_{H}^{\mathrm{T}}, \mathbf{I}_{2}=-\pi \omega \mathbf{Q}_{H} \mathbf{D}^{2} \mathbf{Q}_{H}^{\mathrm{T}} \text { and } \mathbf{D}=\operatorname{diag}(0,1, \ldots, H) ;
$$

- $\mathbf{S}_{\mathrm{nl}}(\tilde{\mathbf{p}})=\left[\int_{I_{t}} q_{k} \mathbf{f}_{\mathrm{nl}}(\mathbf{P q}(t)) d t\right]_{1 \leq k \leq m}$ is computed using the AFT approach described in Sec. 3.1.

The size of this system depends on $N$ so this is more expensive to solve than the temporal problem as $m<<N$, especially in an industrial context.

\subsection{4. $P G D / H B M$ core global algorithm}

Both temporal and spatial problem being properly defined, they are embedded in the global PGD/HBM algorithm which also addresses the convergence criterion issue and additional modes initialization. It is described in Algorithm 1.

First, an error measure $\varepsilon$ is defined (lines 1 and 11). It compares the norm of the residue vector $\mathbf{R}(\mathbf{u}(t))$ where $\mathbf{u}(t)=\mathbf{P Q}_{H} \mathbf{h}_{H}(t, \omega)$ to the norm of the linear stiffness contribution over a period $I_{t}$. This lets us introduce a convergence criterion for the alternated directions algorithm loop (line 4) while Grolet and Thouverez [14] imposed to loop over $k$ three times without checking the convergence. The loop is then broken as soon as the number of iterations $k$ becomes larger than a given value $k_{\max }$ or the error measure $\varepsilon$ becomes smaller than a given limit $\varepsilon_{\max }$ or when the current iteration did not provide enough error reduction, that is, when $\varepsilon>r_{\mathrm{ad}} \varepsilon_{\mathrm{prev}}$, with $r_{\mathrm{ad}}<1$ an arbitrary positive factor, and $\varepsilon_{\text {prev }}$ being the error measured at the end of the previous iteration. 


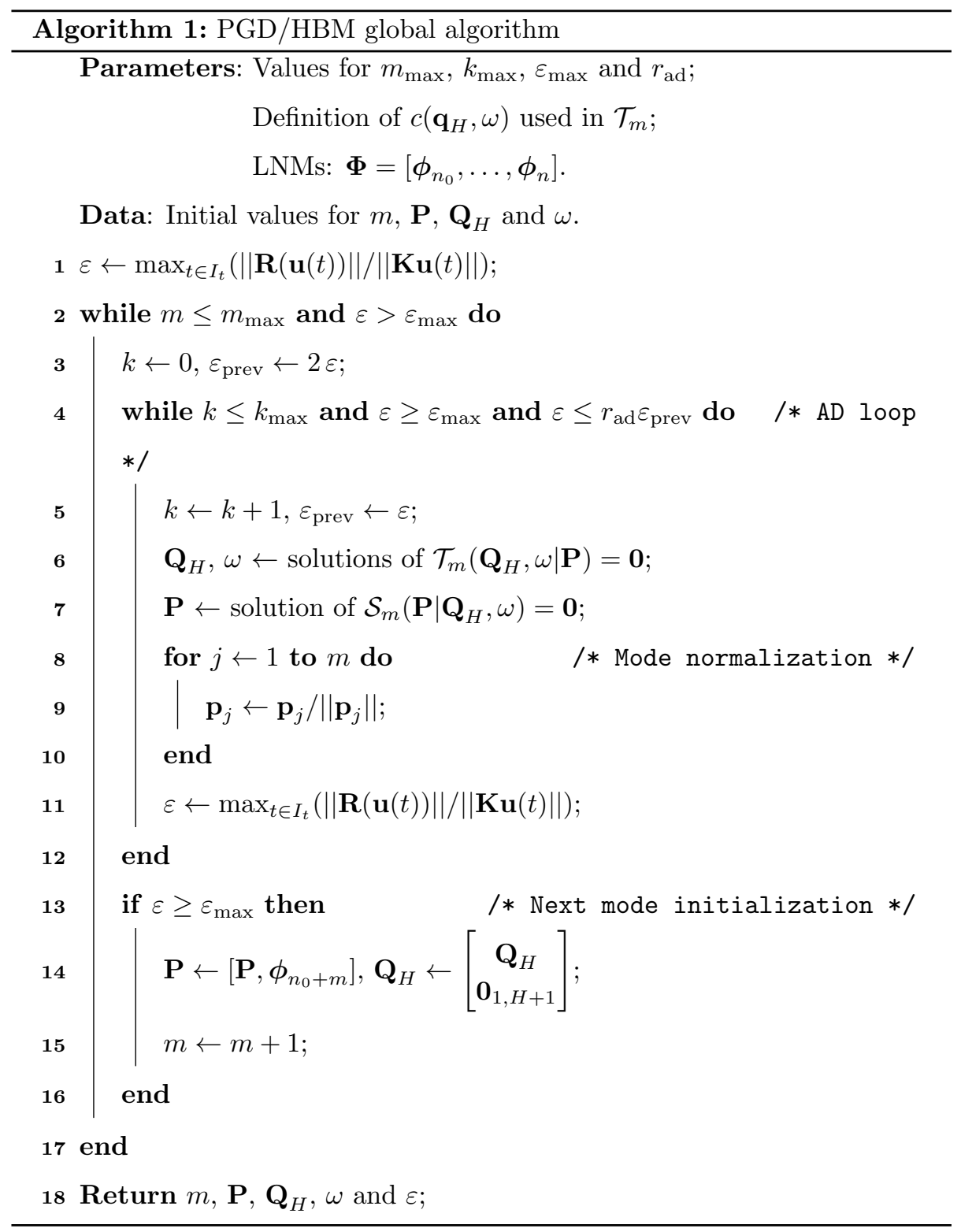


Inside the alternated directions algorithm loop, temporal and spatial problems are solved (lines 6 and 7 respectively). Once a new $\mathbf{P}$ matrix is computed, each spatial mode $\mathbf{p}_{j}$ is normalized as stated in Sec. 3.2.1.

Once the loop is broken, if the convergence criterion $\varepsilon \leq \varepsilon_{\max }$ is not fulfilled, a new PGD mode is added (from line 13): $m$ is increased and a new column is added to $\mathbf{P}$ matrix while a new row is added to $\mathbf{Q}_{H}$ matrix. The choice of these additional values is important as it will greatly influence the convergence of the next resolution loop. Grolet and Thouverez [14] chose to add random values for $\mathbf{Q}_{H}$, and processed the spatial problem before the temporal one. We propose here to process the temporal problem first based on the new spatial mode introduced $\phi_{n_{0}+m}$, with $n_{0}$ being the index of the $n_{0}$-th NNM. Indeed, we do not know much of the relevant temporal information to inject: having to initialize $\mathbf{Q}_{H}$ is a real difficulty. However, the LNMs $\phi_{k}$ solutions of:

$$
\left(\mathbf{K}-\omega_{k}^{2} \mathbf{M}\right) \phi_{k}=\mathbf{0}
$$

220 scheme keeps the number of variables used to describe the branch as small as possible while energy grows and the system behaviour becomes more complex. 
The main interest of this work does not consist in the continuation technique are added along the path. In what follows, the main ingredients of a classical prediction-correction continuation scheme are briefly defined before introducing the choices made to tackle the variable size.

To simplify the notations, we will denote $\mathbf{y}_{n}$ the $n$-th point on the current path. This point gathers the PGD modes as well as the circular frequency information, $\mathbf{P}, \mathbf{Q}_{H}$ and $\omega$ and hence has size $N m+(H+1) m+1$ :

$$
\mathbf{y}_{n} \equiv\left\{\mathbf{P}_{n}, \mathbf{Q}_{H n}, \omega_{n}\right\}
$$

Moreover, the distance $d\left(\mathbf{y}_{n-1}, \mathbf{y}_{n}\right)$ between two consecutive points $\mathbf{y}_{n-1}$ and $\mathbf{y}_{n}$ is defined using separated weights for each variable:

$$
d\left(\mathbf{y}_{n-1}, \mathbf{y}_{n}\right)=\sqrt{\alpha_{P}^{2}\left\|\mathbf{P}_{n}-\mathbf{P}_{n-1}\right\|^{2}+\alpha_{Q}^{2}\left\|\mathbf{Q}_{H n}-\mathbf{Q}_{H n-1}\right\|^{2}+\alpha_{\omega}^{2}\left|\omega_{n}-\omega_{n-1}\right|^{2}}
$$

where $\alpha_{P}, \alpha_{Q}$ and $\alpha_{\omega}$ are strictly positive constants which provide a mean to manage the different orders of magnitudes of the three variables. Each of them can be set to 1 but they can also be adjusted so as to give more or less importance to each of these variables by compensating for the difference in order of magnitudes.

\subsection{Continuation process}

As stated above, a very simple continuation procedure is used. More complex procedures can be found in [32]. Let us assume that $n$ points $\mathbf{y}_{1}, \ldots, \mathbf{y}_{n}$ have already been computed. The next point $\mathbf{y}_{\star}$ is sought using a prediction step which returns $\mathbf{y}_{\star}^{\text {pred }}$; this point is used as an initialization point for the correction step which, in our case, relies on the coupled PGD/HBM method described in Sec. 3.2 with a specific constraint equation $c\left(\mathbf{q}_{H}, \omega\right)=0$ linked to the chosen correction method. The global algorithm is depicted in Algorithm 2.

The prediction is based on a secant method:

$$
\mathbf{y}_{\star}^{\text {pred }}=\mathbf{y}_{n}+\Delta s \frac{\mathbf{y}_{n}-\mathbf{y}_{n-1}}{d\left(\mathbf{y}_{n-1}, \mathbf{y}_{n}\right)}
$$


$\Delta s$ defines "how far" the point is predicted. It is usually adapted along the path: for areas where the solution does not vary a lot, a large value should be used to save computation time; on the contrary, if the solution does vary "rapidly" small values provide better predictions and fewer correction steps. Here, we use a rough adaptation scheme depending on the convergence of the correction step and the error measure value $\varepsilon$ (see Algorithm 1 in Sec. 3.2.4) once a new point was computed: if the correction step did not provide a satisfying new point, $\Delta s$ is decreased by multiplying it by $r_{s}^{\text {div }}<1$ (see line 17) leading further to a new prediction point $\mathbf{y}_{\star}^{\text {pred }}$ closer to $\mathbf{y}_{n}$; if the correction step provided a satisfying point for which the error measure is less than $r_{\varepsilon} \varepsilon_{\max }$ with $r_{\varepsilon}<1, \Delta s$ is multiplied by $r_{s}^{\text {mult }}>1$ (see lines 13 and 14); otherwise, $\Delta s$ is kept constant for the next prediction step.

This secant prediction implies that at least 2 points have already been computed. The first point of the path is taken equal to the LNM associated to the studied NNM with null amplitude. Using notations introduced in Eq. (19) and (20): $\mathbf{y}_{1} \equiv\left\{\phi_{n_{0}}, \mathbf{0}_{1, H+1}, \omega_{n_{0}}\right\}$. To find the second point, we choose to impose the amplitude of the $j$-th dof at $t=0: u_{j}(t=0)=u_{j 0}$ with $u_{j 0} \neq 0$. This prevents the algorithm from returning the null (and trivial) solution. This condition is denoted $c_{2}\left(\mathbf{q}_{H}, \omega\right)$ in Algorithm 2 and defines the constraint equation $c\left(\mathbf{q}_{H}, \omega\right)=0$ of Algorithm 1. The coupled PGD/HBM method can then be applied to find $\mathbf{y}_{2}$ starting from $\mathbf{y}_{1}$.

The correction step consists in applying the PGD/HBM method described in Algorithm 1 with $\mathbf{y}_{\star}^{\text {pred }}$ as a startpoint and using a specific constraint equation ${ }_{275} c\left(\mathbf{q}_{H}, \omega\right)$ involved in the time problem $\mathcal{T}_{m}$.

This equation is denoted $c_{p}\left(\mathbf{q}_{H}, \omega\right)$ in Algorithm 2. It defines the correction method and can be freely chosen among the classical possibilities [24]. An arclength correction is used for applications studied in Sec. 5:

$$
c_{p}\left(\mathbf{q}_{H \star}, \omega_{\star}\right)=d\left(\mathbf{y}_{n}, \mathbf{y}_{\star}\right)^{2}-\Delta s^{2}
$$

This condition also involves $\mathbf{P}_{\star}$ which is the last value of spatial modes returned after the spatial problem $\mathcal{S}_{m}$ when processing the current temporal problem $\mathcal{T}_{m}$. 
As said above, these steps are rather classical for a prediction/correction based continuation procedure. The important difference lies in the possibility of increasing the problem dimension when stepping from $\mathbf{y}_{n}$ to $\mathbf{y}_{n+1}$ as the correction step managed by the PGD/HBM method described in Algorithm 1 can add up to $\Delta m$ modes to $\mathbf{y}_{n+1}$ (see line 7 in Algorithm 2 which defines $m_{\max }$ used in Algorithm 1). This leads to continuation points with possibly different sizes which has to be dealt with.

285

The termination criterion in Algorithm 2 (line 6) involves the maximum number of PGD mode $m_{\max \text { tot }}$. This criterion could of course be advantageously replaced by a more meaningful criterion involving the frequency or the amplitude of a given dof for example. 


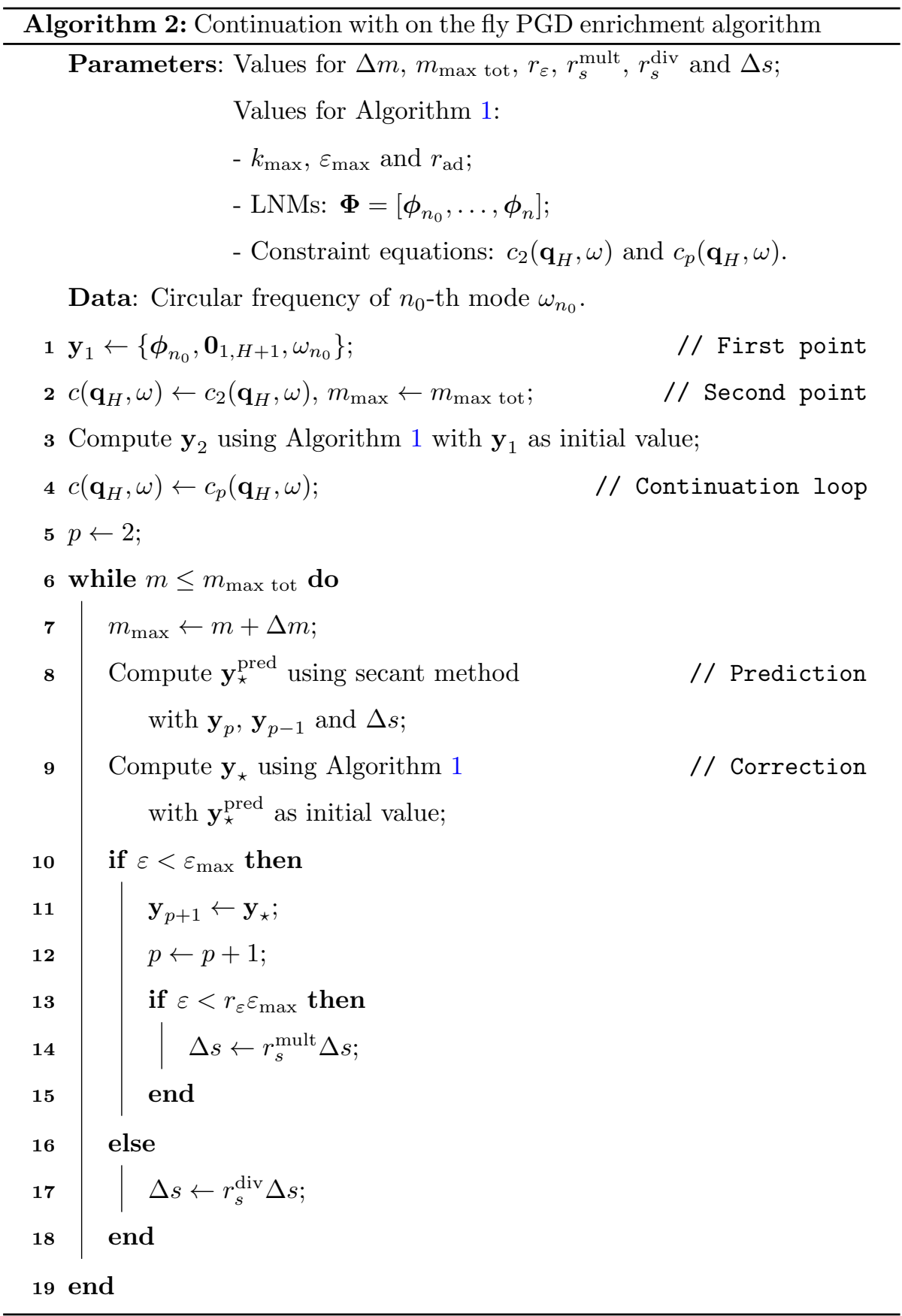


and a spring at its free end. Two different classes of conservative nonlinearities are then added and detailed. These models are generated using MATLAB ${ }^{\circledR}$ 
directly: the need for nonlinear forces $\mathbf{f}_{\text {nl }}$ expression makes it difficult to use the method in conjunction with a commercial FEM software.

Let us define the values of the beam properties: Young modulus $E=$ $210 \mathrm{GPa}$, density $\rho=7800 \mathrm{~kg} \cdot \mathrm{m}^{-3}$, area of the square section $S=910^{-4} \mathrm{~m}^{2}$, second area moment $I=6.7110^{-8} \mathrm{~m}^{4}$ for an in-plane bending and length $L=1 \mathrm{~m}$. The linear stiffness of the spring is $k=410^{4} \mathrm{~N} \cdot \mathrm{m}^{-1}$. The beam is discretized with $N_{e}=20$ elements by FEM with two dof per node: a transverse displacement $u$ and a rotation $\theta$. This leads to $N=40$ dof once the clamping is taken into account. The first eigenvalues of the beam alone and beam plus the linear spring are given in Table 1.

\begin{tabular}{cccccccc}
\cline { 2 - 7 } & $i$ & 1 & 2 & 3 & 4 & 5 & 6 \\
\hline \multirow{2}{*}{$\omega_{i}\left(\operatorname{rad}^{-1}\right)$} & Beam alone & 157 & 988 & 2765 & 5419 & 8959 & 13385 \\
\cline { 2 - 8 } & With spring & 217 & 999 & 2769 & 5420 & 8960 & 13386 \\
\hline
\end{tabular}

Table 1: First eigenvalues of the clamped-free beam alone and with the linear spring attached to the free end.

Both studied cases are illustrated in Fig. 1. In the first case a polynomial nonlinearity is chosen by introducing a cubic stiffness $k_{\mathrm{nl}}$ to the spring. The second case examines a unilateral contact problem: the spring has a linear stiffness but there is a gap $g$ between the free end of the beam and the spring. The parameters required by Algorithm 2 are given in Table 2 for both studied

(a)

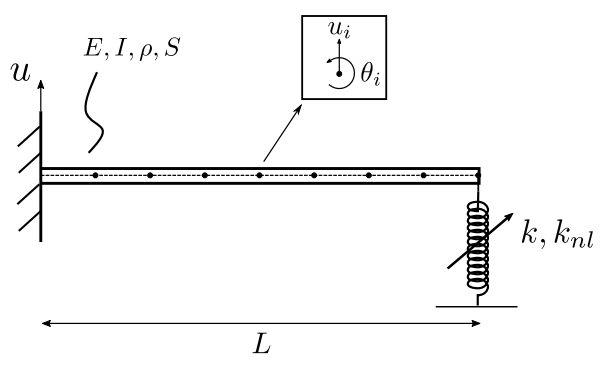

(b)

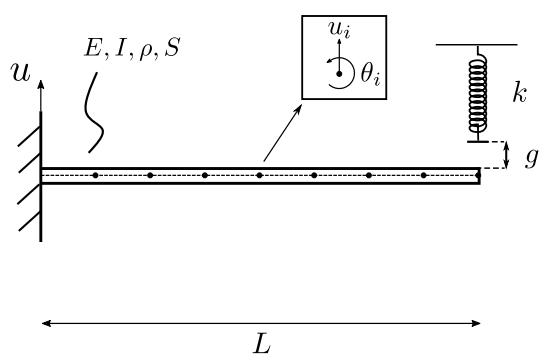

Figure 1: Euler-Bernouilli cantilever beam (a) with a transverse cubic spring at dof 1 (b) with a transverse gap between dof 1 and the linear spring. 
cases.

\begin{tabular}{|c|c|c|c|c|c|c|}
\hline & & & & & & \\
\hline & $\Delta m$ & $m_{\max \text { tot }}$ & $r_{\varepsilon}$ & $r_{s}^{\text {mult }}$ & $r_{s}^{\mathrm{div}}$ & $\Delta s$ \\
\hline \multirow{3}{*}{$\begin{array}{c}\text { Cubic spring case } \\
\text { Contact case }\end{array}$} & 1 & 10 & 0.1 & 1 & 0.5 & 1.5 \\
\hline & 5 & 10 & 0.5 & 1.07 & 0.5 & 1 \\
\hline & $k_{\max }$ & $\varepsilon_{\max }$ & $r_{\mathrm{ad}}$ & \multicolumn{2}{|c|}{$\mathbf{y}_{2}: u_{1}(t=0)$} & $\left\{\alpha_{P}, \alpha_{q}, \alpha_{\omega}\right\}$ \\
\hline \multirow{2}{*}{$\begin{array}{c}\text { Cubic spring case } \\
\text { Contact case }\end{array}$} & 10 & $10^{-3}$ & 0.8 & \multicolumn{2}{|c|}{$10^{-2} \mathrm{~m}$} & $\left\{1,10^{-1}, 10^{-4}\right\}$ \\
\hline & 10 & $10^{-3}$ & 0.8 & \multicolumn{2}{|c|}{$510^{-4} \mathrm{~m}$} & $\left\{0,10^{-6}, 1\right\}$ \\
\hline
\end{tabular}

Table 2: Algorithm 2 required parameters.

\subsection{Bending beam with a cubic spring at its free end} the beam, generating localized curvature inversions near the free end. Kerschen qualitatively gives similar results for another cantilever beam with cubic spring in $[19]$. 
(a)

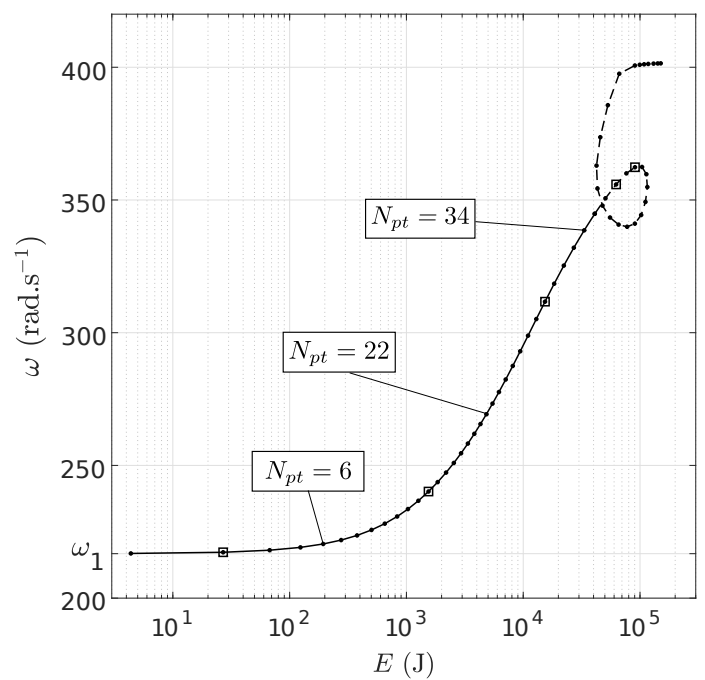

(b)

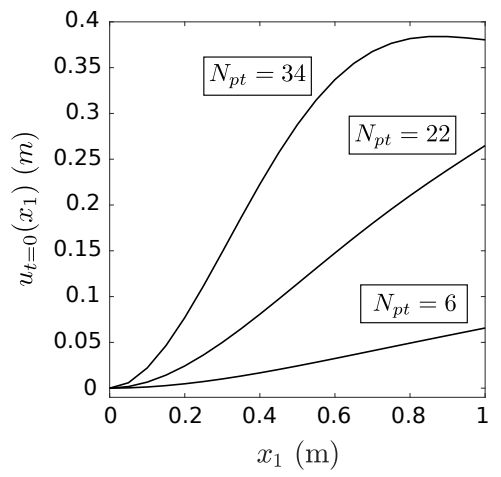

Figure 2: Beam with cubic spring - (a) Main branch of NNM1 in a FEP and (b) its mode shape at null velocity points for three continuation points. Squares: solution points where a PGD mode is added.

The error measure $\varepsilon$ along the path is depicted along with the number of PGD modes on Fig. 3. Its evolution is consistent with the evolution of $m$ : PGD modes are added when $\varepsilon$ reaches the limit value $\varepsilon_{\max }$ which, in turns, decreases the error. Moreover Fig. 2 shows the PGD modes are gradually added along the branch as expected with a smooth cubic nonlinearity. Note that the error for the first point $N_{\mathrm{pt}}=1$ is not displayed as it is null and cannot appear in a logarithmic scale.

Fig. 4 provides an analysis of PGD spatial modes $\mathbf{p}_{j}$. PGD modes globally keep their shape through continuation. An interesting feature proper to the presented method is the presence of PGD modes which involve the participation of several LNMs. Such a "combined mode" is here the PGD mode 3, which gathers contributions of the first 6 LNMs. With such an approach, high order LMNs can be taken into account in a reduced range of PGD mode shapes: here 4 PGD modes are sufficient to describe a system behaviour which involves at 
(a)

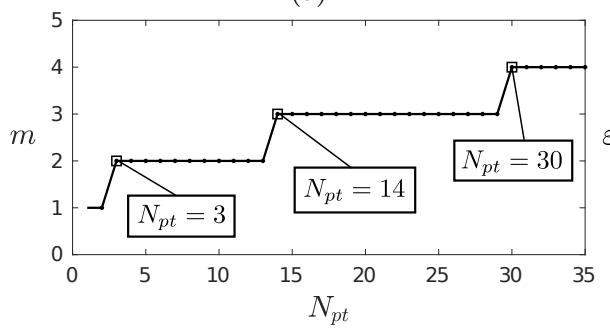

(b)

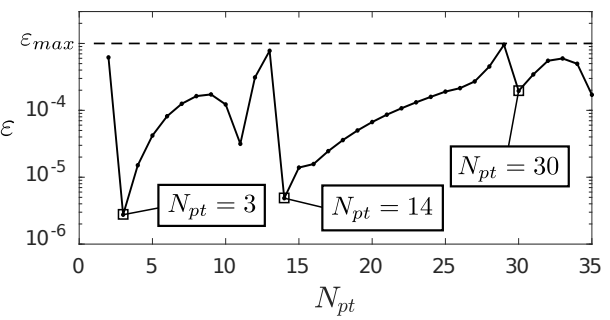

Figure 3: Beam with cubic spring - (a) Number of PGD modes $m$ and (b) error measure $\varepsilon$ against point index $N_{\mathrm{pt}}$.

least 6 LMNs.
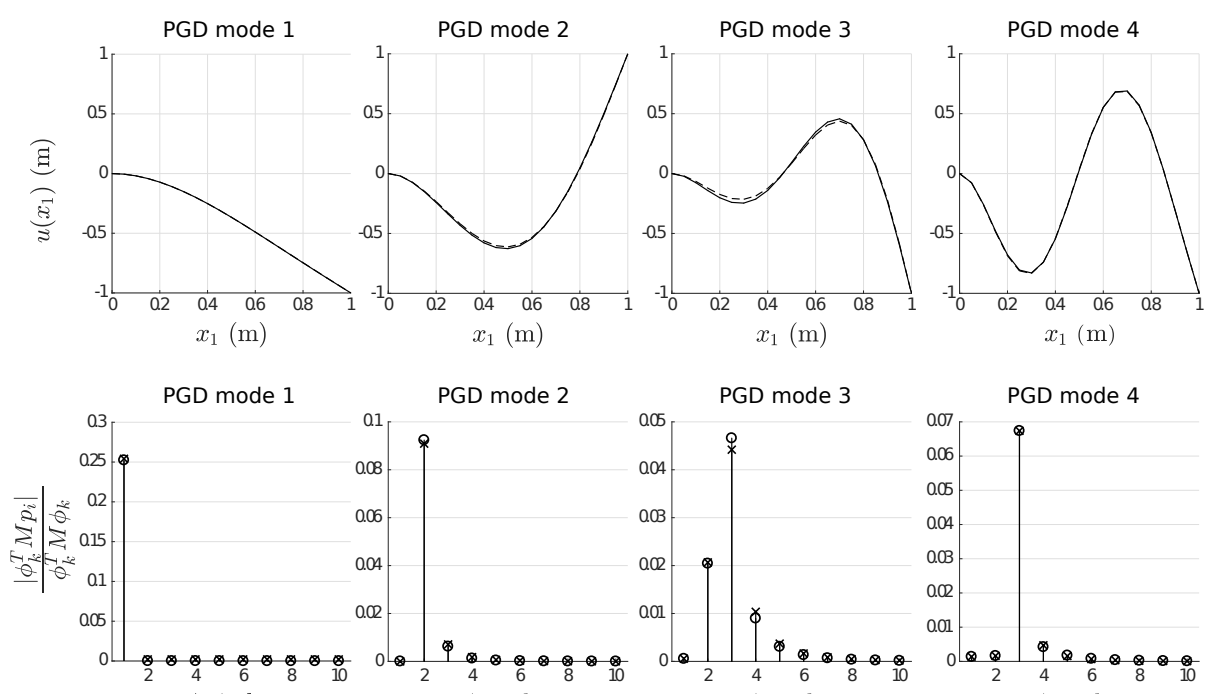

$\phi_{k}$ index

$\phi_{k}$ index

$\phi_{k}$ index

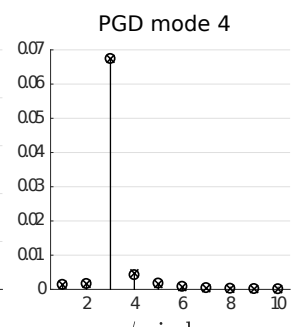

Figure 4: Beam with cubic spring - Analysis of PGD spatial modes. First row: PGD mode shapes. Normalization is made at the maximum deflection point. Dotted line: shape when the mode is first introduced; Solid line: shape at the end of the branch $\left(N_{\mathrm{pt}}=35\right)$. Second row: Participation factors of LNMs in each PGD mode. Crosses: factors when the mode is introduced; Circles: factors at the end of the branch $\left(N_{\mathrm{pt}}=35\right)$.

The temporal information is plotted on Fig. 5. The significant variations of the temporal part through the MNL branch are expected as the previously described spatial part does not significantly change. Otherwise only odd harmonics are not null because of the cubic nature of the nonlinearity. As a global 
trend high order harmonics have less influence: all the amplitudes $\left|a_{k}\right|$ are submillimetric from $k=11$, for all the PGD modes. The PGD mode 1 behaviour is the simplest as it stays mainly led by the fundamental cosine. Other PGD modes have a richer harmonic behaviour with shared influences of $\left|a_{1}\right|,\left|a_{3}\right|$, $\left|a_{5}\right|$ and $\left|a_{7}\right|$. This complex harmonic spectrum is partly due to the fact that PGD mode shapes are combinations of LMNs. All these qualitative interpretations also highlight the growing complexification of the beam behaviour and the growing influence of high harmonics as energy grows.
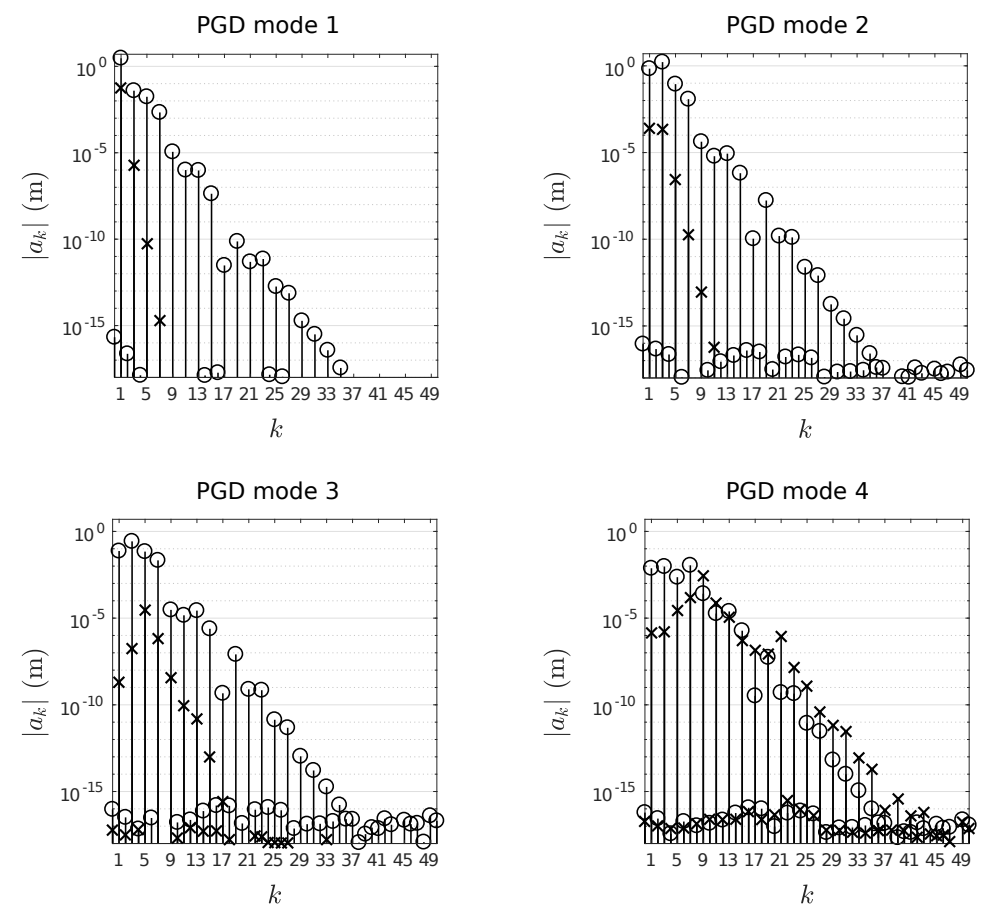

Figure 5: Beam with cubic spring - Amplitudes of (cosine) Fourier coefficients of PGD modes. Crosses: coefficients when the mode is first introduced; Circles: coefficients at the end of the branch $\left(N_{\mathrm{pt}}=35\right)$.

Finally the main objectives of the method are met: to describe a NNM branch with a few variables, to keep the number of PGD modes as small as possible by incrementing $m$ only when the convergence criterion is not fulfilled, and obviously to retrieve all physical data belonging to the case under study. 
In the next section the method is tested on a non polynomial nonlinearity.

\subsection{Bending beam with a unilateral contact}

In this example the underlying linear system is the Euler-Bernoulli cantilever beam. Its modal properties are given in the first row of Table 1. The unilateral contact is modelled as follows:

$$
\mathbf{f}_{\mathrm{nl}}(\mathbf{u})=\left\{\begin{array}{l}
{\left[k\left(u_{1}(t)-g\right) \mathbf{0}_{N-1}\right]^{T}, \text { if } u_{1}(t)>g} \\
\mathbf{0}_{N}, \text { otherwise }
\end{array}\right.
$$

with $g=2 \mathrm{~cm}$. This law is kept non smooth and the contact is quite hard as $k / \frac{3 E I}{L^{3}}=94.56 \%$ with $\frac{3 E I}{L^{3}}$ the spring rate of the cantilever beam taken alone. A similar contact problem from the stiffness point of view is a contact between this beam and another identical one. Looking for periodical solutions for this class of problem usually requires a wide range of harmonics so the arbitrary value $H=70$ is chosen. The consistency of this value is shown a posteriori in Fig. 9.

NNM1 branch displayed in Fig. 6 is built with only 6 PGD modes and 199 solution points for the set of parameters given in Table 2. On the FEP given on Fig. 6 , from $N_{\mathrm{pt}}=1$ to $N_{\mathrm{pt}}=21$, NNM1 is exactly equal to LNM1 - a pure fundamental cosine - as the beam does not touch the spring yet. Then a transition zone occurs for $22 \leq N_{\mathrm{pt}} \leq 85$ where 5 PGD modes are added on 5 close points. Finally the rest of the branch is computed. Note that two internal resonances extracted from two others simulations are drawn with dotted lines. These data are obtained by manually modifying the parameters of Table 2 at the beginning or through the simulation. Analogous FEPs of related contact systems are studied by Moussi and Cochelin [33, 34] for example. 


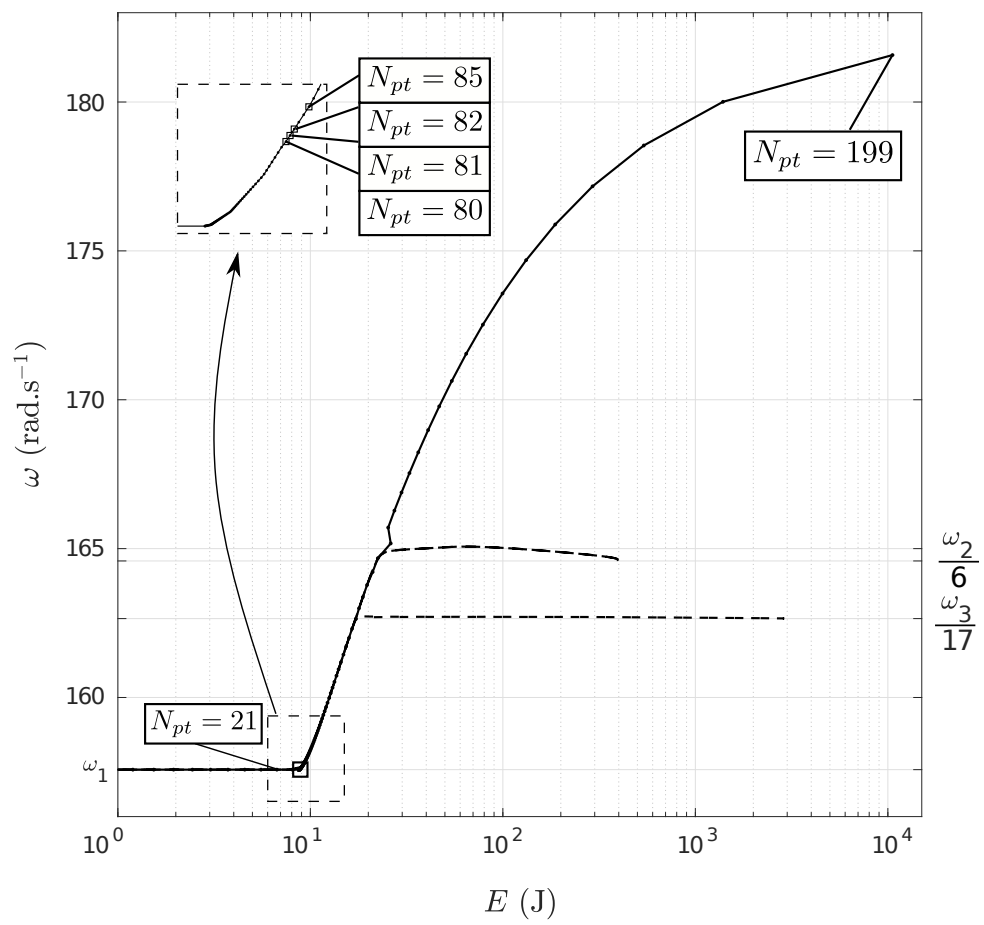

Figure 6: Beam with unilateral contact - NNM1 main branch (solid) and internal resonances (dotted) in a FEP. Squares: points where PGD modes are added.

In this example again the error measure $\varepsilon$ follows the evolution of $m$ as shown on Fig. 7. Here 5 PGD modes are required to significantly decrease the error. Other simulations with different continuation parameters add these 5 modes in one single point when it is allowed by using $\Delta m=5$. Note that by contrast with the cubic spring case all the PGD modes needed to build the branch are added in the same narrow area in the FEP. This shows that describing sharp or gradual changes of the system behaviour by the PGD/HBM continuation scheme with on the fly enrichment is feasible. 
(a)

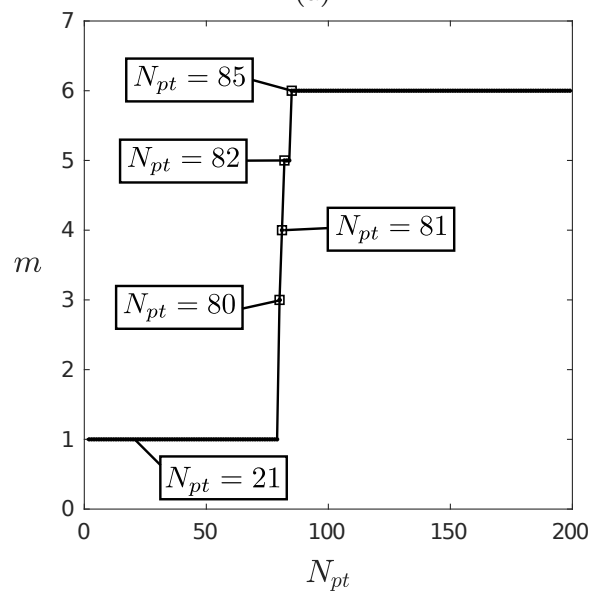

(b)

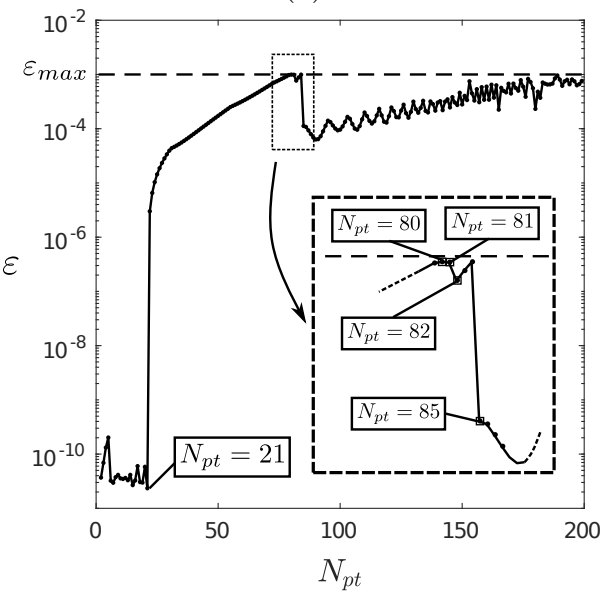

Figure 7: Beam with unilateral contact - (a) $m$ and (b) residue $\varepsilon$ against $N_{\text {pt. Dotted line: }}$ convergence threshold.

As shown on Fig. 8, for this problem PGD mode shapes do not significantly vary except the two last modes. The fifth PGD mode shape strongly changes between $N_{\mathrm{pt}}=82$ and $N_{\mathrm{pt}}=199$, mostly because of LNM4 participation. However its final shape is about to be reached at $N_{\mathrm{pt}}=85$, when PGD mode 6 is introduced, and PGD mode 5 does not evolve a lot from this point. The last PGD mode has remarkable coupling with high order LNMs. It condenses in a single mode a lot of modal information which illustrates the economical benefits of the presented method. 

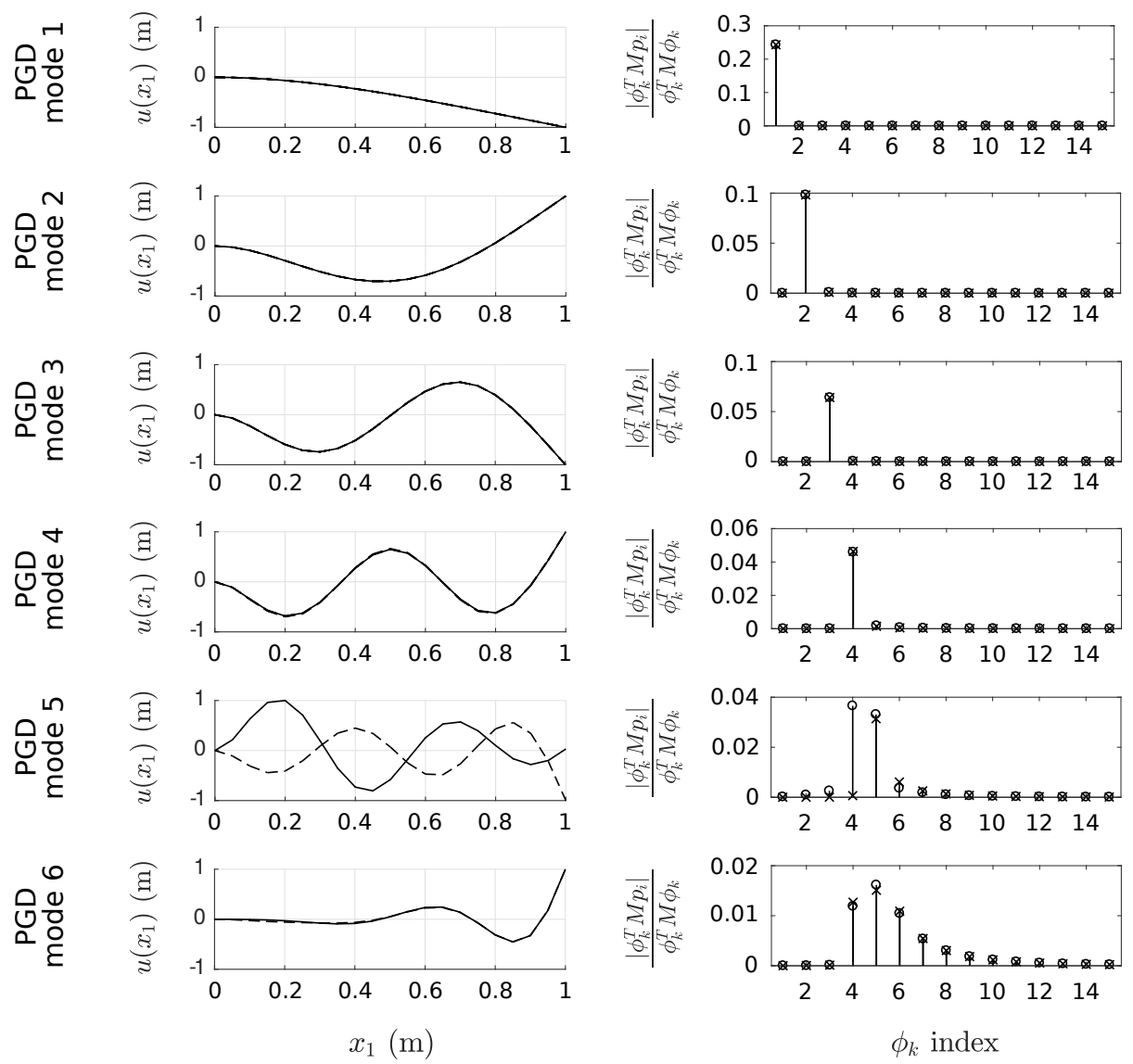

Figure 8: Beam with unilateral contact - Analysis of PGD spatial modes. Left column: PGD mode shapes. Normalization is made at the maximum deflection point. Dotted line: shape when the mode is first introduced; Solid line: shape at the end of the branch $\left(N_{\mathrm{pt}}=199\right)$. Right column: Participation factors of LMNs in each PGD mode. Crosses: factors when the mode is introduced; Circles: factors at the end of the branch $\left(N_{\mathrm{pt}}=199\right)$.

The harmonic decomposition associated to each PGD mode is given on Fig. 9. The temporal part is complex as the contact issue generates a lot of important high harmonics but global trends can be considered. First a predominant cosine in PGD mode 1 drives the linear behaviour at the beginning of the range, then the amplitude of the whole harmonic set increases as energy grows. The free flight of the beam during a period can help to explain why low frequency harmonics are always significant through the continuation. Note that 
non negligible static coefficients $a_{0}$ appear because of the static term introduced in the nonlinear vector $\mathbf{f}_{\mathrm{nl}}(\mathbf{u})$.
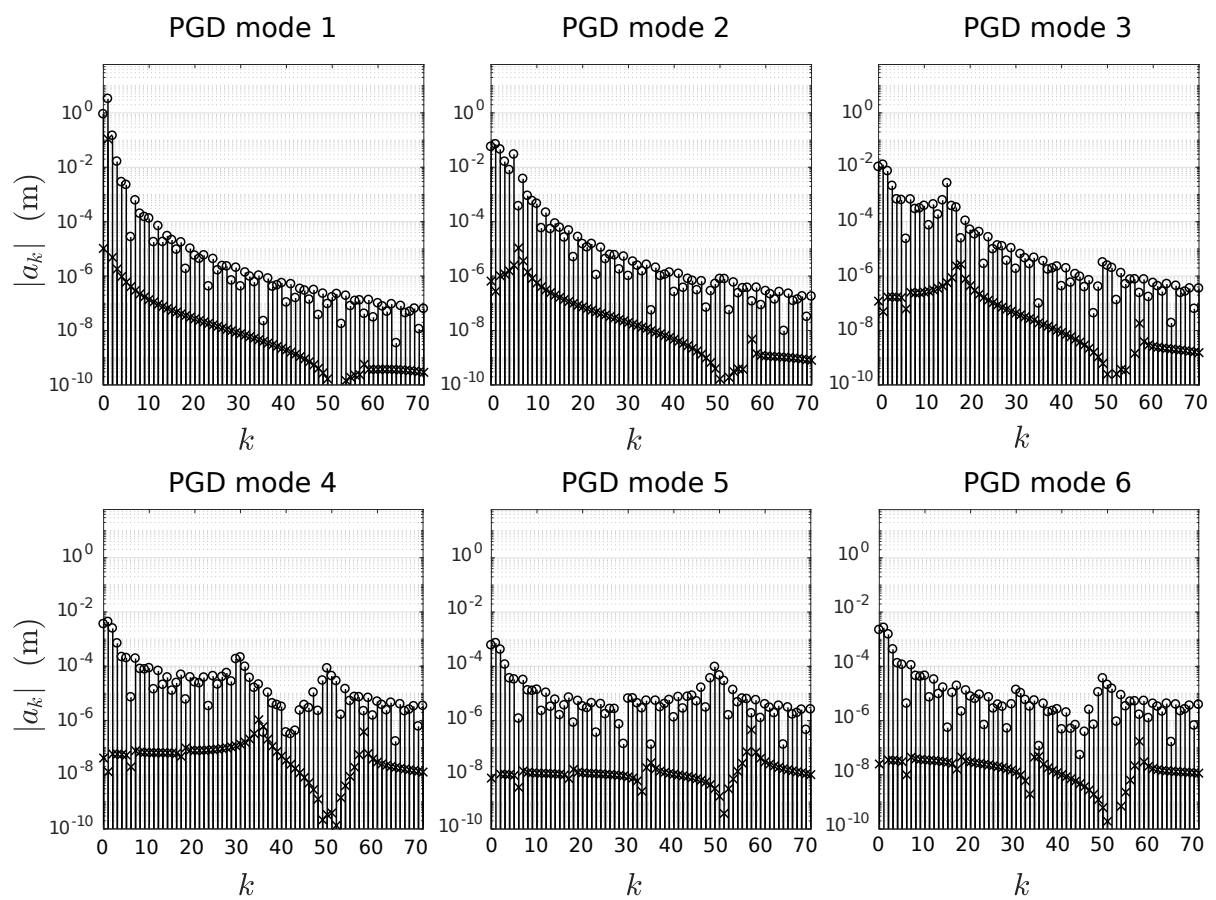

Figure 9: Beam with unilateral contact - Amplitudes of (cosine) Fourier coefficients of PGD modes. Crosses: coefficients when the mode is first introduced; Circles: coefficients at the end of the branch $\left(N_{\mathrm{pt}}=199\right)$.

\subsection{Numerical considerations}

The main objective of reduced model approaches is reached as few descriptors are processed and stored with the presented approach, as shown in Table 3.

A classical HBM continuation requires $N \times(H+1)$ descriptors by solution point whereas the implemented PGD/HBM continuation only needs $m \times(N+$ $H+1$ ) ones. Obviously calculating a solution point via PGD/HBM is relevant

440 if and only if $m \times(N+H+1)<N \times(H+1)$. An easy proof gives the necessary condition for the PGD/HBM method to be more interesting than a classical HBM approach: $m<H+1$. In other words, the PGD/HBM method is useful for problems which require a large number of harmonics. 


\begin{tabular}{|c|c|c|c|c|c|c|}
\hline \multicolumn{6}{|c|}{ PGD/HBM } & HBM \\
\hline \multirow{2}{*}{$\begin{array}{l}\text { Cubic spring } \\
\text { case }\end{array}$} & $\begin{array}{l}m=1 \\
(2 \mathrm{pts})\end{array}$ & $\begin{array}{l}m=2 \\
(11 \mathrm{pts})\end{array}$ & $\begin{array}{l}m=3 \\
(16 \mathrm{pts})\end{array}$ & $\begin{array}{l}m=4 \\
(6 \mathrm{pts})\end{array}$ & Total & \\
\hline & 184 & 2013 & 4384 & 2190 & 8771 & 71435 \\
\hline \multirow[t]{2}{*}{ Contact case } & $\begin{array}{l}m=1 \\
(79 \mathrm{pts})\end{array}$ & \multicolumn{2}{|c|}{$\begin{array}{r}2 \leq m \leq 5 \\
(5 \mathrm{pts})\end{array}$} & $\begin{array}{l}m=6 \\
(115 \mathrm{pts})\end{array}$ & Total & \\
\hline & 7268 & \multicolumn{2}{|c|}{2007} & 62905 & 71971 & 406159 \\
\hline
\end{tabular}

Table 3: Comparaison between the number of descriptors needed by PGD/HBM $-m(N+$ $H+1)+1$ by point - and classical HBM $-N(H+1)+1$ by point - for both examples.

In both examples the saving of variables with respect to a classical HBM can be assessed with the ratio $1-\frac{m \times(N+H+1)}{N \times(H+1)}$. The PGD reduction is very efficient, with $88 \%$ and $82 \%$ less variables for the cubic spring and the contact cases respectively.

We now focus on the underlying numerical solver and its iteration data when the subproblems are solved. The trust-region algorithm used requires function and abscissa tolerances - set on $10^{-9}$ here - and allows to set a maximum number of iterations $N_{\text {ite max }}$. The value $N_{\text {ite } \max }=100$ is set but this limit is never reached (max. 54 iterations for the spatial problem of the cubic spring case, when PGD mode 4 is about to be added). In Table. 4 are given mean and maximum numbers of solver iterations for temporal and spatial problems through the continuation, for both examples.

\begin{tabular}{ccccc}
\cline { 2 - 5 } & & $\begin{array}{c}\text { Mean } \\
\text { iteration number }\end{array}$ & $\begin{array}{c}\text { Standard } \\
\text { deviation }\end{array}$ & $\begin{array}{c}\text { Maximum } \\
\text { iteration number }\end{array}$ \\
\hline \multirow{2}{*}{ Cubic spring case } & $\mathcal{T}_{m}$ & 6.23 & 4.89 & 22 \\
\cline { 2 - 5 } & $\mathcal{S}_{m}$ & 16 & 10.92 & 54 \\
\hline \multirow{2}{*}{ Contact case } & $\mathcal{T}_{m}$ & 2.18 & 1.53 & 16 \\
\cline { 2 - 5 } & $\mathcal{S}_{m}$ & 9.77 & 8.68 & 49 \\
\hline
\end{tabular}

Table 4: Mean, standard deviation and maximum of iteration numbers for $\mathcal{T}_{m}$ and $\mathcal{S}_{m}$ problems, through the continuation.

As expected the spatial problem is heavier and the given maxima are reached when a PGD mode is about to be added. $N_{\text {ite max }}$ could be an interesting 
control parameter for bigger problems as one can keep it very small and save computation time by "converting" convergence issues into new PGD modes or a reduced arclength $\Delta s$.

Another performance indicator is the CPU time. The total computational time spent to build NNM1 of the cubic spring case was approximately 380s with the PGD/HBM approach, against 10400s for a classic HBM, that is about 27 times less. Computations used the same continuation scheme, 36 solutions points were found in both cases and branches were computed up to $E_{\max } \approx$ $4.110^{4} \mathrm{~J}$; they were run on a desktop computer with MATLAB ${ }^{\circledR}$ R2017a, 32 GB of RAM and an Intel ${ }^{\circledR}$ Core i7 CPU.

Finally we compare the numerical efficiency of Algorithm 1 when using the proposed algorithm and when processing the spatial problem $\mathcal{S}_{m}$ first. As explained in Sec. 3.2.4, when $\mathcal{S}_{m}$ is solved before $\mathcal{T}_{m}$, the lack of temporal data to initialize the spatial problem is filled by using random values for $\mathbf{Q}_{H}$. Hence, uniformly distributed random numbers between 0 and 1 are used to initialize $\mathbf{Q}_{H}$. The cubic spring case is computed with exactly the same parameters except the random new PGD modes. In the non-random case, 35 attempts are 475 made to compute the 35 solution points: line 17 in Alg. 2 is never called. To compare the result, among 25 draws of the random case we focus on the number of converged points computed with 35 attempts, the maximum energy reached $E_{\max }$ and the number of iterations data of all the draws. The iteration number of the failed attempts are counted into the iteration number of the next converged point. The results are given in Table 5 .

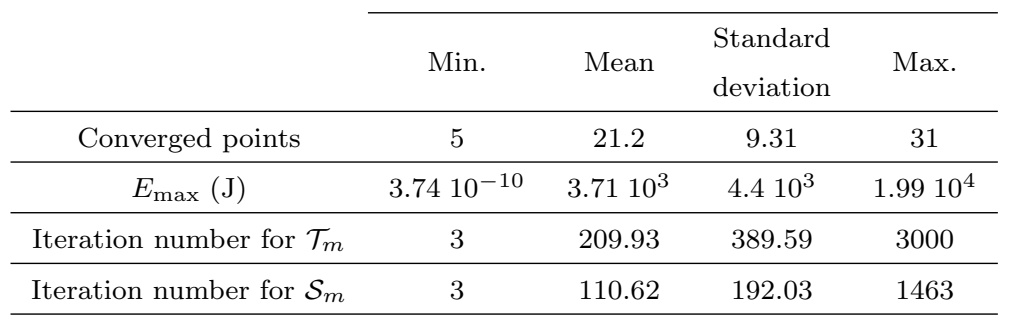

Table 5: Mean, standard deviation and extrema of simulation features for 25 random draws. 
Unlike the non-random computation, there are always failed attempts. The number of computed iterations is far bigger. In particular the temporal problem is heavily penalized by the random coefficients. Otherwise no point reaches the final energy of the non-random branch. Some draws stay at a very low level of energy and 24 out of 25 draws have solutions points which go back into the NNM branch. This shows initializing new modes with random values leads to non robust predictions.

In addition to these points, the main advantage of the presented non-random method against the random variant is the reproducibility of its results.

\section{Conclusion}

The computation of NNMs using a PGD/HBM continuation technique with on the fly enrichment derived in this paper has been presented and successfully applied on two classical nonlinear examples with either smooth or non smooth nonlinearities. The only limitation of the method is to have access to nonlinear forces value $\mathbf{f}_{n l}(\mathbf{u})$ at some specific points $\mathbf{u}$. A significant decrease of the number of needed variables has been noticed when compared with a classical HBM approach; this demonstrates that NNM branches can be described in a highly reduced manner. The PGD modes obtained provide relevant physical data as spatial initializations are the LNMs of the system. The numerical aspects developed here are highly adaptable as many parameters are let free ( $c f$. Table 2), giving a certain flexibility to the algorithm.

The presented approach could be used to process bigger structures with different kinds of nonlinearities. It could be interesting for systems which present many contact issues for example. Variants of the algorithm could then be considered in order to alleviate the cost of the spatial subproblem by using a progressive PGD method which only addresses a spatial problem when a PGD mode is added. On the fly impoverishment of the PGD modes set could also be considered so as to keep it as small as possible.

Finally, future prospects could include the computation of damped NNM or 
forced responses of a structure.

\section{Acknowledgements \& Declaration of interest}

This research did not receive any specific grant from funding agencies in the public, commercial, or not-for-profit sectors.

Declarations of interest: none.

\section{References}

[1] G. Kerschen, M. Peeters, J. Golinval, A. Vakakis, Nonlinear normal modes, part i: A useful framework for the structural dynamicist, Mechanical Systems and Signal Processing 23 (1) (2009) 170-194. doi:10.1016/j.ymssp. 2008.04 .002 .

[2] D. Laxalde, F. Thouverez, J.-J. Sinou, Dynamics of a linear oscillator connected to a small strongly non-linear hysteretic absorber, International Journal of Non-Linear Mechanics 41 (8) (2006) 969-978. doi: 10.1016/j.ijnonlinmec.2006.09.002.

[3] R. Rosenberg, On nonlinear vibrations of systems with many degrees of freedom, Vol. 9 of Advances in Applied Mechanics, Elsevier, 1966, pp. 155 -242. doi:10.1016/S0065-2156(08)70008-5.

[4] S. Shaw, C. Pierre, Normal modes for non-linear vibratory systems, Journal of Sound and Vibration 164 (1) (1993) 85-124. doi:10.1006/jsvi.1993. 1198.

[5] L. Renson, G. Kerschen, B. Cochelin, Numerical computation of nonlinear normal modes in mechanical engineering, Journal of Sound and Vibration 364 (2016) 177-206. doi:10.1016/j.jsv.2015.09.033.

[6] M. Peeters, R. Viguié, G. Sérandour, G. Kerschen, J.-C. Golinval, Nonlinear normal modes, part ii: Toward a practical computation using numerical 
continuation techniques, Mechanical Systems and Signal Processing 23 (1) (2009) 195-216. doi:10.1016/j.ymssp.2008.04.003.

[7] C. Joannin, B. Chouvion, F. Thouverez, J.-P. Ousty, M. Mbaye, A nonlinear component mode synthesis method for the computation of steadystate vibrations in non-conservative systems, Mechanical Systems and Signal Processing 83 (2017) 75-92. doi:10.1016/j.ymssp.2016.05.044.

[8] R. J. Kuether, M. S. Allen, A numerical approach to directly compute nonlinear normal modes of geometrically nonlinear finite element models, Mechanical Systems and Signal Processing 46 (1) (2014) 1-15. doi:10/ f5wk9d.

[9] C. Sombroek, P. Tiso, L. Renson, G. Kerschen, Numerical computation of nonlinear normal modes in a modal derivative subspace, Computers \& Structures 195 (2018) 34-46. doi:10/gdnbvq.

[10] F. Chinesta, P. Ladeveze, E. Cueto, A short review on model order reduction based on proper generalized decomposition, Archives of Computational Methods in Engineering 18 (4) (2011) 395-404. doi : 10.1007/s11831-0119064-7.

[11] F. Chinesta, A. Ammar, E. Cueto, Recent advances and new challenges in the use of the proper generalized decomposition for solving multidimensional models, Archives of Computational Methods in Engineering 17 (4) (2010) 327-350. doi:10.1007/s11831-010-9049-y.

[12] F. Chinesta, A. Leygue, F. Bordeu, J. V. Aguado, E. Cueto, D. Gonzalez, I. Alfaro, A. Ammar, A. Huerta, Pgd-based computational vademecum for efficient design, optimization and control, Archives of Computational Methods in Engineering 20 (1) (2013) 31-59. doi:10.1007/s11831-0139080-x.

[13] A. Nouy, A priori model reduction through proper generalized decomposition for solving time-dependent partial differential equations, Computer 
Methods in Applied Mechanics and Engineering 199 (2324) (2010) 16031626. doi:10.1016/j.cma.2010.01.009.

[14] A. Grolet, F. Thouverez, On the use of the proper generalised decomposition for solving nonlinear vibration problems, in: ASME 2012 International Mechanical Engineering Congress and Exposition, no. 45202, American Society of Mechanical Engineers, 2012, pp. 913-920. doi: 10.1115/IMECE2012-87538.

[15] T. L. Hill, A. Cammarano, S. A. Neild, D. A. W. Barton, Identifying the significance of nonlinear normal modes, Proceedings of the Royal Society A: Mathematical, Physical and Engineering Science 473 (2199) (2017) 20160789. doi:10/f93fxs.

[16] D. Laxalde, F. Thouverez, Complex non-linear modal analysis for mechanical systems: Application to turbomachinery bladings with friction interfaces, Journal of Sound and Vibration 322 (4-5) (2009) 1009-1025. doi:10/dzk672.

[17] L. Renson, G. Kerschen, Nonlinear Normal Modes of Nonconservative Systems, Springer New York, New York, NY, 2013, pp. 189-202. doi: 10.1007/978-1-4614-6570-6_17.

[18] S. Shaw, C. Pierre, Non-linear normal modes and invariant manifolds, Journal of Sound and Vibration 150 (1) (1991) 170-173. doi:10.1016/0022460X (91) 90412-D.

[19] G. Kerschen, Computation of Nonlinear Normal Modes through Shooting and Pseudo-Arclength Computation, Springer Vienna, Vienna, 2014, pp. 215-250. doi:10.1007/978-3-7091-1791-0_5.

[20] G. Haller, S. Ponsioen, Nonlinear normal modes and spectral submanifolds: existence, uniqueness and use in model reduction, Nonlinear Dynamics 86 (3) (2016) 1493-1534. doi:10.1007/s11071-016-2974-z. URL https://doi.org/10.1007/s11071-016-2974-z 
[21] P. Sundararajan, S. T. Noah, Dynamics of forced nonlinear systems using shooting/arc-length continuation method-application to rotor systems, Journal of Vibration and Acoustics 119 (1) (1997) 9-20. doi: $10.1115 / 1.2889694$.

[22] M. Urabe, Galerkin's procedure for nonlinear periodic systems, Archive for Rational Mechanics and Analysis 20 (2) (1965) 120-152. doi:10.1007/ $\mathrm{BF} 00284614$.

[23] M. Urabe, A. Reiter, Numerical computation of nonlinear forced oscillations by galerkin's procedure, Journal of Mathematical Analysis and Applications 14 (1) (1966) 107-140. doi:10.1016/0022-247X (66) 90066-7.

[24] A. H. Nayfeh, D. T. Mook, Nonlinear Oscillations, Wiley, 1995.

[25] E. Sarrouy, J.-J. Sinou, Non-linear periodic and quasi-periodic vibrations in mechanical systems - on the use of the harmonic balance methods, in: Advances in Vibration Analysis Research, InTech, 2011. doi:10.5772/ 15638.

[26] R. Seydel, From Equilibrium to Chaos, Practical Bifurcation and Stability Analysis, Elsevier, 1988.

[27] T. M. Cameron, J. H. Griffin, An alternating frequency/time domain method for calculating the steady-state response of nonlinear dynamic systems, Journal of Applied Mechanics 56 (1) (1989) 149-154. doi: $10.1115 / 1.3176036$.

[28] E. Sarrouy, O. Dessombz, J.-J. Sinou, Stochastic study of a non-linear selfexcited system with friction, European Journal of Mechanics - A/Solids 40 (2013) 1-10. doi:10.1016/j.euromechsol.2012.12.003.

[29] N. Coudeyras, J.-J. Sinou, S. Nacivet, A new treatment for predicting the self-excited vibrations of nonlinear systems with frictional interfaces: The constrained harmonic balance method, with application to disc brake 
squeal, Journal of Sound and Vibration 319 (3-5) (2009) 1175-1199. doi: $10.1016 / j \cdot j s v \cdot 2008.06 .050$.

[33] B. Cochelin, Numerical computation of nonlinear normal modes using HBM and ANM, Springer Vienna, Vienna, 2014, pp. 251-292. doi:10.1007/ 978-3-7091-1791-0_6.

[34] E. Moussi, S. Bellizzi, B. Cochelin, I. Nistor, Nonlinear normal modes of 635

[30] E. J. Doedel, Auto: A program for the automatic bifurcation analysis of autonomous systems, Congressus Numerantium 30 (1981) 265-284.

[31] T. L. Hill, A. Cammarano, S. A. Neild, D. J. Wagg, Out-of-unison resonance in weakly nonlinear coupled oscillators, Proceedings of the Royal Society of London A: Mathematical, Physical and Engineering Sciences 471 (2173). arXiv:http://rspa.royalsocietypublishing.org/ content/471/2173/20140659.full.pdf, doi:10.1098/rspa.2014.0659. URL http://rspa.royalsocietypublishing.org/content/471/2173/ 20140659

[32] E. L. Allgower, K. Georg, Introduction to Numerical Continuation Methods, Springer-Verlag, 2003.

a two degrees-of-freedom piecewise linear system, Mechanical Systems and Signal Processing 64 (2015) 266-281. doi:10.1016/j.ymssp.2015.03. 017. 